\section{The 31st Annual Meeting of the J.B. Johnston Club and the 23rd Annual Karger Workshop}

The 2011 meeting of the J.B. Johnston Club and Karger Workshop will be held immediately before the annual meeting of the Society for Neuroscience on Thursday, November 10 (the Karger Workshop), and Friday, November 11 (the regular JBJC meeting), 2011. Both the Karger Workshop and the regular JBJC meeting will take place at the Embassy Row Hotel in Washington, D.C., USA.

This year's Karger Workshop, made possible by the continuing support of Karger Press, was organized by Kara E. Yopak and is entitled 'The Nervous System of Cartilaginous Fishes.' The Karger Workshop features seven invited talks by speakers who will present new insights into the structure, function, development and evolution of the nervous system in diverse cartilaginous fishes. On the following day, the program for annual JBJC meeting will consist of 16 talks submitted by JBJC members selected by the JBJC Program Committee (Kenneth Catania, Ann Butler, and Barbara Finlay) plus a presentation by this year's invited Karger Speaker, Dr. John Montgomery. Additional information and the final schedule of talks will be mailed to JBJC members before the meeting, and will be posted on the JBJC web site (www.jbjclub. org).

\section{Karger Workshop: \\ The Nervous System of Cartilaginous Fishes}

\section{Organized by: Kara E. Yopak (University of Western}

Australia, Crawley, W.A., Australia)

Speakers giving presentations at the 2011 Karger Workshop are listed below. The final schedule of talks will be sent to the membership prior to the meeting and will be available at the registration desk during the meeting.

\section{The Cerebellum and Cerebellum-Like Structures of Cartilaginous Fishes}

John C. Montgomery, University of Auckland, Auckland (New Zealand)
The Neuroecology of Cartilaginous Fishes: Sensory Strategies for Survival

Shaun P. Collin, University of Western Australia, Crawley, W.A. (Australia)

Connecting Life-History and Brain Development in Chondrichthyans: Using Phylogenetic Comparative Analysis to Examine the Relationship between Maternal Investment and Brain Growth

Christopher Mull, Simon Fraser University, Burnaby, B.C. (Canada)

\section{Contributions of Developmental Studies in the Dogfish Scyliorhinus canicula to the Brain Anatomy of Elasmobranchs}

Isabel Rodríguez-Moldes, University of Santiago de Compostela, Santiago de Compostela (Spain)

\section{A Review of Elasmobranch Electroreception: Sawfish as a Case Study}

Barbara Wueringer, University of Western Australia, Crawley, W.A. (Australia)

\section{Neuroanatomy of the Elasmobranch Forebrain}

Michael Hofmann, University of Bonn (Germany), and Glenn Northcutt, University of California San Diego, Calif. (USA)

\section{More than Meets the Eye: Scaling of the Optic Tectum across Cartilaginous Fishes}

Kara E. Yopak, University of Western Australia, Crawley, W.A. (Australia), and Thomas J. Lisney, University of Alberta, Edmonton, Alta. (Canada)

\section{J.B. Johnston Club Meeting Abstracts}

Abstracts for talks scheduled for the 2011 annual meeting of the J.B. Johnston Club are listed in alphabetical order by presenting author. The final schedule of talks will be sent to the membership prior to the meeting and will be available at the registration desk during the meeting. This year's Karger Invited Speaker will

\begin{tabular}{ll}
\hline KARGER & @ 2011 S. Karger AG, Basel \\
Fax +41613061234 & \\
$\begin{array}{l}\text { E-Mail karger@karger.ch } \\
\text { www.karger.com }\end{array}$ & $\begin{array}{l}\text { Accessible online at: } \\
\text { www.karger.com/bbe }\end{array}$
\end{tabular}


be Dr. John Montgomery. The title of Dr. Montgomery's talk will be: 'Do Cerebellum and Cerebellum-Like Structures Share the Same Functional Algorithm?'

\section{Flight Dynamics and Spatial Memory in Echolocating Bats}

Jonathan R. Barchi ${ }^{a}$, Andrea M. Simmons ${ }^{b}$

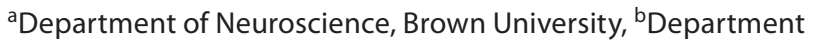
of Cognitive, Linguistic and Psychological Sciences,

Brown University, Providence R.I., USA

(barchi@brown.edu)

Echolocating bats use an active sensory system that is limited in its effective range to $5-10 \mathrm{~m}$ by atmospheric attenuation of $\mathrm{ul}-$ trasonic signals. Despite this limitation, they navigate deftly in three dimensions and travel as far as several kilometers along consistent routes between roosting and foraging sites. In addition, the use of echolocation while hunting means these bats must optimize the partitioning of echolocation calls between navigation and prey detection. This suggests an important role for spatial memory in long-range navigation and while foraging. To investigate this, individuals and groups of bats of several species were recorded in the laboratory and in the field using thermal cameras and arrays of 4-24 ultrasonic microphones (depending on location). In laboratory experiments, bats (Eptesicus fuscus) were tested for correlates of learning and recall in a cluttered flight environment. From these data, bat position and echolocation call behavior were reconstructed and analyzed for path stereotypy, call rate, and behavioral signatures of an internal spatial map using custom software implemented in MATLAB. Both laboratory and field data are consistent with the idea that bats construct an internal representation of space that is tightly coupled to their echolocation behavior and flight dynamics.

\section{Brain Scaling across Vertebrates: Conservation and Variation}

Christine J. Charvet, Richard B. Darlington, Barbara L. Finlay

Department of Psychology, Cornell University, Ithaca, N.Y., USA (charvetcj@gmail.com)

A conserved pattern of allometric scaling of major brain components related to a conserved pattern of onset and duration of neurogenesis in mammalian brain regions was found in an extended group of mammals [Finlay \& Darlington, 1995; Reep et al., 2007], and more recently in sharks [Yopak et al., 2010]. These studies also showed an interesting and 'conserved' relative independence of the olfactory bulb and associated limbic components from the more regular scaling of the rest of the brain. Here we extend the same analysis of the six major brain region volumes to 253 vertebrates, now including ray-finned fishes, amphibians, and birds. We also compare the allometry of 4 telencephalic subdivisions (i.e., medial, dorsal, lateral-ventral pallial divisions and striatum) in birds and in mammals. The basis for our delineation of telencephalic divisions in birds and mammals follows the claustroamygdalar hypothesis that postulates that the dorsal ventricular ridge of birds is homologous to the claustrum and amygdala of mammals.

The extension of the data set confirmed the basic findings of the phylogenetically bracketing, shark-to-mammal earlier analysis, but also produced some interesting surprises. The telencephalon, diencephalon, mesencephalon, cerebellum and medulla volumes strongly co-vary and are highly predictable. The telencephalon and cerebellum are hyper-allometric with respect to every other brain region across vertebrates. In contrast, the olfactory bulb volume varies much more independently than the rest of the brain, particularly in birds, and large-brained mammals. For example, the diencephalon volume varies by a factor of 2 , while the olfactory bulb volume varies by a factor of 26 in a $10-11 \mathrm{~cm}^{3}$ vertebrate brain. Grade shifts are common between vertebrate classes, the most common observation being an increase of hyperallometry in the telencephalon or cerebellum in a derived group with respect to a stem group. One interesting exception is that the relative volume and allometric scaling of the cerebellum is reduced in amphibians compared to fishes, mammals and birds. Within the telencephalon of birds and mammals, an interesting distinction in scaling emerges: birds selectively enlarge the lateral and ventral pallial divisions while mammals enlarge their dorsal and medial pallial divisions, but in such a precisely inverse relationship that the entire telencephalon scales identically in the two groups. With the exception of the variable size of the olfactory bulb and limbic system, which in separate taxa often aligns with a nocturnal or diurnal niche or its equivalent, it was difficult to find any behavioral or niche assignment (e.g., carnivory), which across vertebrates accounted for any additional variance in the size of these gross brain regions.

We suggest that the size of gross brain regions at the crossvertebrate level is the result of fundamental scaling and computational constraints - for example, the cerebellum might be best conceived as a structure permitting prediction and error correction, not 'motor skills.' Similar to the developmentally conserved vertebrate body and brain plan, overall vertebrate brain scaling is the palette from which species differences may be drawn.

Supported by NSF grant DBI0848612 and NIH grant 1F32HD067011-01A1.

\section{The Role of the Zebra Finch Cerebellum in Cognition and Song}

\section{Lainy Day, Rory Spence, Barney Schlinger, Josh Hamer, Gerald Stinson, Matthew DiGiusto}

University of Mississippi, Biology Department, University, Mass., University of California, Los Angeles, Calif., USA

(lainyday@olemiss.edu)

Flourens famously demonstrated almost 200 years ago that removal of the cerebellum (CB) led to impaired motor coordination in pigeons and chickens. Since these first rudimentary studies, CB research in birds has been overshadowed by mammalian 
studies despite the fact that avian CBs are relatively larger when compared to mammals with similar sized cerebrums. The recent studies in humans and other mammals have shown that the $\mathrm{CB}$ plays a role not only in coordination, but also a prominent role in cognitive tasks with and without motor output such as abstract reasoning, working memory, procedural aspects of spatial learning, speech rhythm and intonation, and emotional control. While sufficient research has been done to assert that the $\mathrm{CB}$ plays a role in motor coordination in all vertebrates, there have been only a handful of studies in non-mammals that have investigated cognitive/emotion functions of the cerebellum. Using a songbird model, the zebra finch, we have been examining the role of the avian cerebellum in cognition. We have developed a novel spatial task to track cognitive deficits. To track any motor deficits that accompany cognitive deficits, we have also developed a method to track subtle motor deficits after cerebellum lesions. Interestingly, obvious motor deficits are temporary after various CB lesions; yet, subtle deficits in motor coordination may persist after the initial recovery period. A method for measuring these deficits, akin to the rotor-rod for rats, did not exist in birds until the development of our apparatus. In our studies we have found, that like mammals, motor deficits in the zebra finch are relatively subtle even with large lesions. We have additionally demonstrated that, like the mammalian cerebellum, the zebra finch cerebellum is involved in spatial cognition and song production. This evidence along with that from other labs showing that the cerebellum of teleost fish is involved in spatial cognition, classical conditioning of eye retraction, and emotional responses, suggests that the function of the vertebrate cerebellum in spatial, emotion, and learned vocalizations is conserved in vertebrates.

\section{An Expensive Matter: Brain Scaling with a Constant Energetic Cost per Neuron and Its Implications for Brain Evolution}

\author{
Suzana Herculano-Houzel
}

Instituto de Ciências Biomédicas, Universidade Federal do Rio de Janeiro, Instituto Nacional de Neurociência Translacional, Brazil

(suzanahh@gmail.com)

The recent analysis of numbers of neuronal and non-neuronal cells, most of which are glial, in the brains of primates and rodents [Herculano-Houzel et al., 2006, 2007] indicates that the numeric ratio of glial to neuronal cells $(\mathrm{G} / \mathrm{N})$ increases monotonically with decreasing neuronal density across structures and species of rodents and primates alike, in line with the notion that larger neuronal size is accompanied by larger numbers of glial cells per neurons.

The larger $\mathrm{G} / \mathrm{N}$ ratio has been suggested to reflect a presumably larger energetic requirement of larger neurons. This would be expected from the increased membrane surface area and presumably increased number of inputs and firing rate of larger neurons, given that the energetic requirements of neurons have been estimated to relate mostly to the restoration of membrane poten- tials altered by synaptic transmission [Atwell and Laughlin, 2001]. However, it remains to be determined whether larger neurons indeed have increased synaptic activity and larger energy use.

Using published data on the glucose consumption by the brain of six mammalian species ( 3 rodents and 3 primates, including humans; Karbowski, 2007) and their numbers of neurons [Herculano-Houzel et al., 2006, 2007; Azevedo et al., 2009] the scaling of total brain energy consumption with its number of neurons was examined. Contrary to the expected, the estimated average glucose use per neuron varies only $40 \%$ across the species, and does not vary together with neuronal density $(p=0.9380)$. Remarkably, and although different cellular scaling rules apply to rodent and primate brains, total glucose use by the brain is a linear function of the number of neurons in the brain across the 6 species $(\mathrm{p}<$ 0.0001 ), at an average cost of about $6 \mathrm{kCal} /$ day per billion brain neurons.

These findings indicate that, contrary to the decreasing energy cost of other cell types as they increase in size, neurons have an energy budget that is relatively invariant across species, brain sizes, and neuronal sizes, which implies that mechanisms must be in place that adjust firing rate and avoid excessive synaptic activity. Synaptic homeostasis and plasticity may therefore be direct consequences of a limiting and size-invariant, fixed neuronal energy budget.

The non-decreasing energetic cost of larger neurons also has important implications for evolution. The greater the caloric needs of a species, the greater the time that must be spent on feeding, if feeding capacity does not increase at the same rate as body size. A simple model of scaling of energetic intake and expenditure with increasing body mass and number of neurons shows that feeding exclusively on a raw diet makes metabolism a limiting factor in primate evolution such that a trade-off between body mass and number of brain neurons imposes itself. Thus, larger numbers of neurons come at a cost of either longer feeding times, smaller $\mathrm{M}_{\mathrm{BD}}$, or a combination of both. This trade-off is a likely explanation for why the largest primates, the great apes, are not also those endowed with the largest brains, and provides strong support for the hypothesis that the advent of cooking foods was a major factor in the rapid evolutionary increase in the number of brain neurons in the genus Homo.

\section{The Pig and the Puddling Claustrum}

\author{
John Irwin Johnson, Kenneth J. Buchanan, Brian A. Fenske, \\ Ayesha S. Yalamarthy
}

Department of Radiology, Division of Anatomy, Michigan State University, East Lansing, Mich., USA

(johnij@aol.com)

The claustrum is an apparently homogeneous group of neurons adjacent to the rhinal sulcus in most mammals. It appears to arise from cortical cells during fetal development, and has been reported to be surrounded by a scattered set of cortical cells that may have been displaced by the claustral expansion [Mathur et al., 2009]. The claustrum forms reciprocal connections with almost 
all areas of cortex. While its functions are generally unknown, its implication in the neural support of consciousness has been suggested [Crick and Koch, 2005].

In humans and other anthropoid primates, the claustrum appears as a distinctive group of cells in a thin lamina enclosed between the striatum and the insular cortex. This confinement gave the structure is anatomical name. In our ongoing survey of the claustrum and insular cortices of the major branches of the mammalian radiation [Buchanan and Johnson, 2011] we were intrigued to find that in some marsupials and xenarthrans, most of the claustral cells were not claustrated at all, but formed a globular structure anterior to the striatum. In most carnivores, the claustrum appears to erupt from the top of the confined striato-cortical interval to form a sharply pyramidal structure reaching into the white core of a neighboring cortical gyrus. In larger brains, those of a zebra, a llama, and several cetaceans [Butti and Hof, 2010], claustral points expand into supervening gyral cores. In cetaceans outlying points form isolated beads. Even in the anthropoid primates, the claustral cells escape their enclosure and form a large mass ventral to the striatum, adjacent to the anterior commissure, amygdala and endopiriform regions.

Pigs show unusual elaboration in claustral morphology. An anterior ball, as in the marsupials and xenarthrans, morphs into a laminar shape more caudally; further posteriorly, claustral cells invade the core of an overlying gyrus forming a carnivorelike pyramid; then a great mass of claustral cells pours, as it were, out the posterior end of the claustral entity, to form a large lobulated or striated nuclear structure subjacent to the ectosylvian gyrus. Also, trailing tails of claustrum spill and trickle out superiorly, inferiorly, medially and laterally. On the basis of the shape of the pig claustrum, and of the distinctive shapes seen in other brains, we make three suggestions: (1) Aggregates of claustral cells do not have an intrinsic shape based on their internal connectivity and organization, rather the grouping is sculpted by the structure of the surrounding neural entities: fiber tracts, nuclei, and cortex. In the course of their development, the claustral cell bodies 'puddle' in available spaces. (2) The massive pools of pig claustral cells, particularly the huge posterior loboid, render pigs potential optimal subjects for studies of claustral anatomy, interconnection, activity and function. (3) The occurrence of an exuberantly elaborated claustrum in the pig brain co-occurs with a cortical olfactory system also of marked size and complexity; this may be related to a role of olfactory specialization in determining the size, shape, position, and function of the claustrum.

\section{References}

Buchanan KJ, Johnson JI: Diversity of spatial relationships of the claustrum and insula in branches of the mammalian radiation. Ann New York Acad Sci 2011;1225(S1):E30-E63.

Butti C, Hof PR: The insular cortex: a comparative perspective. Brain Struc Function 2010; 214:477-493.

Crick FC, Koch C: What is the function of the claustrum? Phil Trans R Soc B 2005;360:1271-1279.

Mathur BN, Caprioli RM, Deutch AY: Proteomic analysis illuminates a novel structural definition of the claustrum and insula. Cereb Cortex 2009;19:2372-2379.

\section{Data Transformations Affect Conclusions on Brain Part Co-Evolution and Encephalization in Primates}

\author{
Louis Lefebvre
}

Department of Biology, McGill University, Montréal, Qué., Canada

(louis.lefebvre@mcgill.ca)

Several comparative studies of encephalization and brain part evolution offer conflicting hypotheses on the conditions and processes that are behind variation in the size of neural structures. In primates, almost all the studies use the same Stephan, Baron \& Frahm database, but transform these raw data into a 'bewildering deluge' of indices. The 4 main studies on brain part evolution each use a different transform, and the 26 large-scale studies on primate encephalization use 26 different measures of brain and neocortex size. Before we conclude anything about the variables that drive primate encephalization and the rules that guide brain part co-evolution, we first need to understand how different transformations affect the data. In this study, I show that the 26 encephalization measures and the 4 brain part transformations have conflicting and often paradoxical effects on the data: the same brain structure can yield quasi identical patterns of variation across primates with some transformations, or completely opposite or completely unrelated ones with other transformations.

\section{Novel Intensity-Dependent Re-Coding of Submillisecond Spike-Timing Differences}

\author{
A.M. Lyons-Warren, T. Kohashi, S. Mennerick, B.A. Carlson \\ Washington University in St. Louis, Department of Biology, \\ St. Louis, Mo., USA
}

(warrena@wusm.wustl.edu)

Detection of submillisecond spike timing differences is a fundamental problem in neuroscience. Time-coding has been studied in timing 'experts' such as echolocating bats, weakly electric fish and barn owls, as well as in the sound localization circuits of other birds, reptiles, and mammals. While the specific anatomical specializations differ, all of these systems utilize similar algorithms, including a combination of time comparator neurons, delay lines and inhibition, to convert peripheral sensory events into a new coding system. The electrosensory circuit used by mormyrid fishes to process communication signals similarly relies on a central comparison of small spike timing differences generated at the periphery. Based on anatomical connections, Friedman and Hopkins proposed a novel delay-line anticoincidence detection mechanism for detecting small spike timing differences. According to this model, small cells in the anterior exterolateral nucleus (ELa) receive variably-delayed excitation and precisely-timed inhibition corresponding to the start and end of a signal. The timing of these inputs depends on the length of the axonal delay to that particular small cell and the duration of the stimulus. The longer the stimulus duration, 
the greater the probability that delayed excitation will arrive before inhibition making each individual small cell long-pass tuned. Further, because the length of the excitatory axonal delay varies for each cell, the minimal stimulus duration to elicit a response will also vary. Therefore, the number of active small cells will reflect stimulus duration, thereby recoding a temporal code into a population code. A direct test of this mechanism requires recording from small cells which is extremely technically difficult. However, we used retrograde transport of fluorescent dye from the posterior exterolateral nucleus (ELp) to selectively label small cells in ELa. This is possible because small cell axons are the only afferent projections from ELa to ELp. We recorded extracellular action potentials from these individual labeled axons in response to monophasic positive and negative square pulses varying in duration from 0.01 to $100 \mathrm{~ms}$ at intensities ranging from 0.145 to $110 \mathrm{mV} / \mathrm{cm}$. We found non-duration selective (allpass) responses to low intensity stimuli. Increasing stimulus intensity increased the number of spikes per repetition over the all-pass responses with the greatest increase seen for longer durations (long-pass). In most neurons, further increases in intensity led to the greatest increase in response rate over a narrow range of durations, thereby establishing band-pass tuning. In some neurons, we observed a decreased response to a narrow range of durations (band-stop) at high intensities. Peripheral electrosensory receptors are long-pass tuned to duration with variability in duration tuning thresholds. Therefore, excitatory and inhibitory inputs to a particular small cell, which come from different receptors, can have different response properties. Band-pass tuning occurs only at intensities high enough to recruit both excitation and inhibition. In conclusion we demonstrate a selective increase in coding precision that converts a peripheral population code into a labeled-line code and propose a novel mechanism for stimulus intensity-dependent duration tuning by time comparator neurons in the midbrain of mormyrid fish.

\section{Matching of Inner Ear Sensitivity to Call Frequency in the Coqui Frog}

Peter M. Narins ${ }^{a, b}$, Sebastiaan W. F. Meenderink ${ }^{c}$

aDepartment of Integrative Biology and Physiology,

${ }^{b}$ Department of Ecology and Evolutionary Biology, University

of California Los Angeles, Calif., 'Department of Physics and

Astronomy, University of California Los Angeles, Los Angeles,

Calif., USA

Acoustic communication involves both the generation and the detection of a signal. In the Puerto Rican coqui frog (Eleutherodactylus coqui), it is known that the spectral contents of its calls systematically change with altitude above sea level. We have now used distortion product otoacoustic emissions (DPOAEs) to assess the frequency range over which the inner ear is sensitive. To make these measurements, we use a portable, non-invasive system that is easily deployed in the field. It is found that both the spectral contents of the calls and the inner ear sensitivity change in a similar fashion along an altitudinal gradient. As a result, the call fre- quencies and the auditory tuning are closely matched at all altitudes. We suggest that the animal's body size determines the frequency particulars of the call apparatus and the inner ear. Supported by NIH grant no. DC-00222 and UCLA Academic Senate grant no. 3501 to PMN, and NWO-VENI grant no. 863.08.003 to S.W.F.M.

Figure 1. Scatter plot showing relationship between the frequency at maximum DPOAE amplitude $\left(F_{\operatorname{maxDP}}\right)$ and the dominant Co-note frequency in the call.

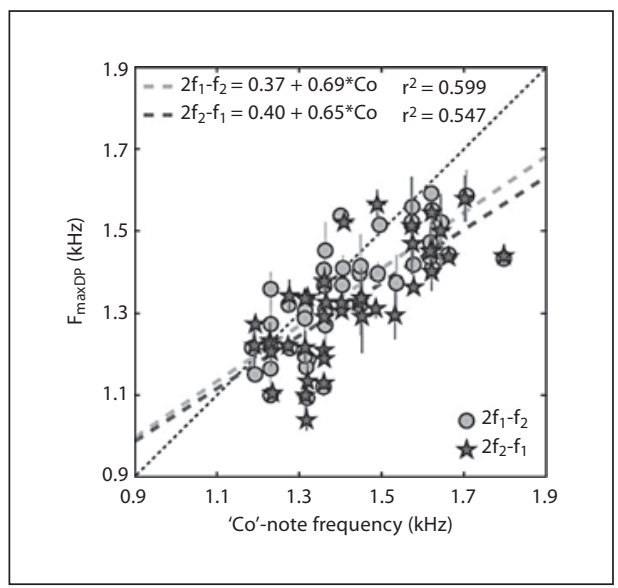

\section{The Relationship between Telencephalon Morphology and Spatial Learning of the Threespine Stickleback Fish (Gasterosteus aculeatus) in Relation to Inferred Ecology}

\author{
Peter J. Park ${ }^{a}$, Ivan Chase ${ }^{b}$, Michael A. Bell ${ }^{c}$ \\ aDepartment of Biology and Chemistry, Nyack College, \\ Nyack, N.Y., Departments of bSociology, 'Ecology and Evolution, \\ Stony Brook University, Stony Brook, N.Y., USA \\ (ppark728@gmail.com)
}

The relationship between telencephalon morphology and spatial learning in relation to inferred ecology and experience was investigated in an Alaskan adaptive radiation of threespine stickleback fish (Gasterosteus aculeatus). Unlike other learning strategies, the extent of spatial learning covaries with the size of a neuroanatomical structure. In Study \#1, we tested the hypothesis that relative size of the telencephalon (adjusted for overall brain size) is larger in benthic-feeding stickleback (benthics) that occupy spatially complex habitat compared to open-water planktivores (limnetics) from habitats with less structure. Field-preserved and lab-bred samples were studied. Contrary to expectations, fieldpreserved benthic populations did not consistently have larger relative telencephalon sizes than limnetic populations. However, the telencephala of benthic populations were more convex laterally than those of limnetics, which may indicate enlargement of the dorsolateral region in benthics. The dorsolateral region of the telencephalon in teleosts is homologous to the tetrapod hippocampus, and greater relative size of the hippocampus is associated 
with superior spatial learning. In contrast to field-preserved fish, there were no differences for telencephalon shape between labbred benthics and limnetics. In addition, relative telencephalon sizes of field-preserved fish were larger than those of lab-bred ones. In Study \#2, spatial learning ability of benthic and limnetic stickleback populations were compared using a T-maze. Benthics exhibited superior spatial learning compared to limnetics. However, lab-bred lacustrine fish from one benthic population performed poorly compared to their field-caught counterparts, suggesting that experience is important for spatial learning. Taken together, these results indicate that compared to limnetics, benthic stickleback are superior spatial learners and possibly possess a larger dorsolateral region of the telencephalon. However, these traits may depend heavily on phenotypic plasticity.

\section{Is the Neuropeptide Kisspeptin a Link between Behavioral and Gonadal Sex Change in the Bluehead Wrasse (Thalassoma bifasciatum)?}

Melissa Slane, John Godwin

Department of Biology, North Carolina State University, Raleigh, N.C., USA

(maslane@ncsu.edu)

The environment plays an essential role in modulating reproductive behavior and physiology in vertebrates. Though neuroendocrine mediation of reproduction is becoming increasingly wellunderstood, the mechanisms by which environmental information is conveyed to the hypothalamic-pituitary-gonadal (HPG) axis are less clear. The kisspeptin (Kiss) system has fairly recently been identified as crucial for normal reproduction in many vertebrates, but its interaction with other neuroendocrine systems requires elucidation. The neuropeptide Kiss is present in most vertebrate species and is expressed in various tissues of the body, including the hypothalamus. In both mammalian and fish models, Kiss mediates reproductive function by activating gonadotropin-releasing hormone $(\mathrm{GnRH})$ neurons. In addition to its important role in puberty and reproduction in gonochoristic fishes and other vertebrates, we hypothesize that Kiss signaling is necessary for socially-induced sex change in sequentially hermaphroditic fishes. We are using the protogynous bluehead wrasse (Thalassoma bifasciatum) as a model to test this hypothesis. T. bifasciatum exhibits female-to-male sex change and male phenotypic change from a nonaggressive, non-courting initial phase (IP) sneaker male to a dominant, courting terminal phase (TP) male. Sex change from a female to a TP male occurs rapidly after a TP male dies or is experimentally removed from a social group. Behavioral changes can begin within minutes and are associated with increased hypothalamic expression of arginine vasotocin (AVT). Gonadal sex change from ovaries to testes takes 7-10 days to complete, and several lines of evidence suggest that changes in GnRH signaling are important in gonadal sex change. We propose that socially-induced changes in AVT signaling influence GnRH neuron activity through effects on the Kiss system. This model is suggested by several recent findings including co-localization of arginine vasopressin (AVP; mammalian homologue of AVT) axons with certain Kiss neurons in mice, identification of Kiss neurons in regions of the hypothalamus where AVT receptors are expressed in teleost fishes, and Kiss activation of $\mathrm{GnRH}$ neurons in mammalian and fish models. Our lab previously cloned $A V T$ in $T$. bifasciatum, and we recently cloned partial cDNAs from one of the Kiss genes, kiss2, and from the Kiss receptor (kiss1r/gpr54). This will allow for determination of Kiss2 and Kiss1r distributions and comparisons with previously characterized distributions of AVT and AVT receptors in the ventral hypothalamus. Potential interactions between AVT/AVP, kisspeptin, and GnRH neuron populations could be critical in transducing social cues into reproductive responses in sex-changing wrasses and possibly an important mechanism mediating social influences on reproduction in vertebrates generally.

\section{Novel Sensory Adaptation in a Rare Cavefish from the Andes \\ Daphne Soares \\ Department of Biology, University of Maryland, College Park, Md., USA \\ (daph@umd.edu)}

Extant cavefishes are natural replicate experiments in adaptation to an extreme environment. Various surface dwelling fish species have independently invaded caves throughout evolutionary time. Thus, the existence of independently derived cavefish forms provides a unique opportunity to examine parallel evolution and convergence. All cavefish ancestors had to outmaneuver and adapt to the harsh constraints imposed by caves and their perpetual darkness. As a result, a suite of unique troglobitic phenotypes have emerged, with loss of pigmentation and eyes being prominent but also, for example, longer and more numerous mechanosensitive neuromasts. Here I examine the cave catfish Astroblepus pholeter. This fish is a member of the Andean catfishes group and inhabits a single cave in Ecuador (Jumandy: $77^{\circ} 47^{\prime} 33^{\prime} \mathrm{W} 0^{\circ} 52^{\prime} 30^{\prime} \mathrm{S}$ ). My results show that this fish species is of special interest because it appears to be the first teleost to have completely lost their neuromasts. Instead, I found that $A$. pholeter's skin is covered with a putative sensory organ. The sensory organ consists of a stiff enamel shaft that emerges from a bulbular base embedded in the skin. The anatomical arrangement is not dissimilar to that observed in hair follicles. Schafer and BuitragoSuárez [2002] found similar organs in the barbels, nasal flap and lips of surface catfishes of the same family (Astroblepus longifilis, $A$. choate and A. rosei) and named them dermal teeth (Odontodes). A. pholeter however, display longer (200 vs. $25 \mathrm{~mm}$ in surface species) and intensely innervated organs all over the head and dorsal portion of the body. Further, surface species of Astroblepids all have neuromasts. One clue for the evolution of this organ comes from the nature of the environment. The Jumandy cave often has high current streams and A. pholeter is only found attached to either the walls or the substrate of the cave. It is possible that neuromasts are overwhelmed with mechanosensory information in the strong current. In the darkness of the cave, A. pholeter may have lost useless neuromasts and evolved mechanosensory organs to detect a spectrum of intensities that neuromasts cannot. 


\section{A Phylogenetic Survey of Morphological Patterns of Superficial Neuromasts in Teleost Fishes}

Song, Jiakun ${ }^{a}, b$, Fan Chunxin ${ }^{a}$, Wang Xiaojie ${ }^{a}$, Zhang Xuguang ${ }^{a}$

anstitute for Marine Biosystem and Neurosciences,

Shanghai Ocean University, China; 'bivision of Fishes,

NMNH Smithsonian Institution, Washington D.C., USA

(jksong@umd.edu, or jksong@shou.edu.cn,or songj@si.edu)

The mechanosensory lateral line receptors have two types, the canal (CNMs) and the superficial neuromasts (SNMs). The SNMs are grouped as three classes in the fishes: the primary superficial neuromasts (pit organs), replacement and accessory of canal neuromasts. However, 'Are they related to phylogenetically evolution or ecologically adaptation?'remains as a question. Systematically survey on the three classes of the SNMs in fish phylogeny has never been conducted.

This study is following a working hypothesis that: the Primary SNMs occur in two patterns. The pit-line pattern, such as in Amia and Gars, a limited number of pit-lines restricted to three regions of head (dorsal surface posterior to the orbits, cheek, and jaws), is a primitive pattern for osteichthyan fishes. The derived non-pit-line pattern(s) include dispersed clusters (or stitches and rows) or reduced lines; these also include the Accessory of CNMs, which should be different in position from the replacement of CNMs. A total of 204 specimens of 184 species represent 41 families from all four teleostean superorders, are examined. Teleost fishes with the primitive pattern include members of the Elopomorpha, Osteoglossomorpha, Clupeomorpha, five groups of Euteleostei: Salmonidae, Argentinoidei, Osmeroidei, Esocae, as well as Gonorynchiformes and Siluriformes of Ostariophysi. The derived non-pit-line pattern(s), included dispersed clusters (or stitches and rows) or reduced lines, occurs in the remaining Ostariophysi (Cypriniformes and Characiformes), Stomiiformes, Aulopiformes and Acanthomorpha of the Euteleostei. Pit-line versus diverse pattern(s) can be used as a criterion of phylogenetic relationships among teleost fishes. Questions of homologues of these patterns in fish groups, their ontogenetic basis and phylogenetic congruency are further discussed.

\section{Bat Wing Sensors Improve Flight Maneuverability \\ S.J. Sterbing-D'Angeloc, M. Chadha ${ }^{a, b}$, C. Chiu ${ }^{b}$, B. Falk ${ }^{b}$, W. Xian ${ }^{b}$, J. Barcelob ${ }^{b}$ J.M. Zook ${ }^{d}$, C.F. Moss ${ }^{a-c}$ \\ aprogram in Neuroscience and Cognitive Science, ${ }^{\mathrm{b}}$ Department of Psychology, 'Institut for Systems Research, University of Maryland, College Park Md., 'Department of Biological Sciences, Ohio University, Athens, Ohio, USA \\ (ssterbin@umd.edu)}

Bats are the only mammals capable of powered flight, and can perform complicated flight maneuvers like tight turns, hovering or perching upside-down. The bat wing membrane is covered with microscopically small hairs, and we hypothesized that these hairs may be involved in sensorimotor flight control by providing aerodynamic feedback. While the existence of these wing hairs has been known for a long time, their actual function during flight has not been experimentally addressed. Here, we present electrophysiological and behavioral data concerning the hairs' functional role as revealed by experiments conducted before and after hair removal. We found that neurons in bat primary somatosensory cortex respond to stimulation of the wing hairs with lowspeed air flow, and that this response is diminished after removal of the hairs. We further found that depilation of different functional regions of the wing membrane alters flight behavior in obstacle avoidance tasks in particular by reducing maneuverability indicated by decreased turn angles. This effect was apparent in both bat species although they have different flight behavior: Eptesicus fuscus is an insectivorous bat, and Carollia perspicillata, a frugi- and nectarivorous species that hovers frequently. Sensory hairs on the wing and their role for flight control have been described for insects. Here we show that also a relatively large animal like the bat shares this feature. Our findings provide the foundation for future biomimetic adaptations of such sensors to improve flight vehicles.

\section{Is There More to Ov than Meets the Ear? Inputs to the Ventromedial Hypothalamus from the Auditory Thalamus and Medial Arcopallium in Songbirds}

\section{J. Martin Wild}

Department of Anatomy, University of Auckland, Auckland, New Zealand

(jm.wild@auckland.ac.nz)

Provided a female songbird is in breeding condition, her hearing conspecific male song, even in the physical absence of that male, can elicit reproductive behavior, specifically the copulation solicitation display (CSD). How this can happen repeatedly and with such short latency is puzzling, in view of the fact that there are no reports of any descending projections from the auditory brainstem upon bulbospinal neurons that might play a role in mediating the CSD. In doves and songbirds it is known that a projection from the interface of the central nucleus of the inferior colliculus (MLd) and the medial part of the intercollicular nucleus (ICo) targets the ventromedial (aka posteromedial) hypothalamic nucleus (VMN or PMH) via peri-ovoidal nuclei [Durand et al., 1992; Zeng et al., 2004]. VMN then projects back upon ICo [Berk and Butler, 1981], thereby providing a loop that in female doves has been suggested to mediate auditory self-stimulation important for the neuroendocrine control of ovulation [Cheng and Zuo, 1994; Cheng and Durand, 2004]. Somewhat similarly in some anuran amphibian species, certain dorsal thalamic nuclei, which receive projections directly from the auditory midbrain torus, have been found to project upon the hypothalamus, where they may also play a role in acoustically mediated gonadotropic function [Neary and Wilczynski, 1986; Allison and Wilczynski, 1991]. Here we report that, in zebra finches and canaries, rostral levels of nucleus ovoidalis (Ov) itself and the nucleus of the tractus ovoidalis (nTOv) both project upon VMN. Ov, which receives a tono- 
topic and probably a topographic projection from MLd, has been proposed as the homologue of the ventral nucleus of the medial geniculate body of mammals, but has thus far been thought to have only ascending projections to the telencephalic auditory Field $\mathrm{L}$ and the striatum. The TOv, in which numerous neurons are embedded, is also reported to project upon Field L, but here we show that it also projects upon VMN. Whether individual Field L-projecting Ov and nTOv neurons also project upon VMN via branched axons is presently unknown.

Injections of a retrograde tracer in $\mathrm{VMN}$ not only labeled Ov and nTOv neurons, but also numerous neurons in the amygdaloid taenial nucleus (ATn) and the adjacent para-taenial area (APTn). Neither of these nuclei is the nucleus taeniae of the amygdala $(\operatorname{Tn} A)$ often referred to in the avian literature, which has been re-named the amygdalohippocampal area (AHi) by Puelles et al. [2007].

It remains to be demonstrated whether the Ov-VMN projection described here is involved in activation of the CSD, but recent studies of Ov in zebra finches and budgerigars [Amin et al., 2010; Brauth et al., 2007] have questioned its supposed simple auditory relay function and allow us to speculate on its possible role in processing vocalizations.

\section{Anatomy of Turtle Middle Ear Cavities and Hindbrain Auditory Circuits}

Katie L. Willis ${ }^{a}$, Catherine A. McCormick ${ }^{b}$, Catherine E. Carr ${ }^{a}$

aDepartment of Biology, Univ of Maryland, College Park, Md.,

${ }^{b}$ Departments Biology and Neuroscience, Oberlin College,

Oberlin, Ohio, USA

(kwillis@umd.edu)

Testudines inhabit a wide range of ecosystems and have many notable morphological features, including a large middle ear cavity. We are developing a fuller picture of the auditory system evolution and organization, by examining both skeletal and neural elements.

Since the connections of the hindbrain auditory nuclei have not been described in detail in turtles [Miller and Kasahara, 1979; Belekhova et al, 1984, 2008; Browner and Marbey, 1988], we labeled the auditory nerve, or injected small amounts of neurobiotin directly into either brainstem or midbrain nuclei of Trachemys scripta elegans or Pseudomys floridana [Willis et al., 2011b]. We identified the three previously described brainstem nuclei: Nucleus Magnocellularis (NM), Nucleus Angularis (NA), and Nucleus Laminaris (NL). NM was located medial and dorsal, near the edge of the medulla, projected across the dorsal midline to terminate in the contralateral NL, and also projected to the ipsilateral NL. NL was located ventral to NM, and consisted of a lamina of vertically-oriented bitufted cells, and horizontally oriented cells dorsal to the lamina. NL projected to the contralateral torus semicircularis (TS). NA also projected largely contralaterally to TS. NA cell types were heterogeneous, falling into two broad categories: multipolar and stubby. NA extended further rostrally and was more ventral and lateral than NM. The superior olive also projected to TS. These projections follow the pattern observed in the other reptiles, including archosaurs.

31st Annual JBJC Meeting and 23rd

Annual Karger Workshop
One hypothesis for the function of the large middle ear cavity of testudines is that it resonates at ecologically important frequencies, enhancing sound perception [Willis et al., 2011a]. Previous studies regarding this cavity have examined this resonance in air [Patterson, 1966; Wever, 1978]. Auditory brainstem responses and laser vibrometry, however, revealed a best sensitivity to underwater sound pressures of $300-500 \mathrm{~Hz}$ [Christensen-Dalsgaard et al., 2010]. The ear was about $10 \mathrm{~dB}$ less sensitive to underwater sound pressures than in air, which in terms of sound intensity showed that thresholds in water are lower than in air and may be indicative of specialization for underwater hearing.

Using micro-CT and micro-MRI scans of more than 20 species of turtles and tortoises, we reconstructed the middle ear cavity digitally using image stacks of the scans, analyzed with Neurolucida (MicroBrightField, Williston, Vt.) by tracing the borders of each cavity and using the volume calculator. The middle ear cavity was a curved ellipsoid that scaled with head size. The exception to this pattern was the Mata Mata, Chelus fimbriatus, which displayed a more hourglass morphology but scaled in the same manner. Middle ear cavities ranged in size from 0.03 to 2.56 $\mathrm{ml}$; head widths ranged from 19-96 mm, and these values varied linearly with each other. By approximating the middle ear cavity as a sphere vibrating underwater, we calculated the best resonance frequencies of the cavities; the lowest was $385 \mathrm{~Hz}$, the highest $1,737 \mathrm{~Hz}$, corresponding to the largest and smallest heads respectively. After separating species by ecology and phylogeny, as well as looking at the distribution as a whole, we found no significant difference in the variation of middle ear cavity volume and head width relationship. This consistency of size and shape, regardless of ecology, supports the hypothesis that testudines had an aquatic origin [Rieppel and Reisz, 1999].

\section{Evolution of the Nervous System Adapts Astyanax to Life in Darkness}

\section{Masato Yoshizawa}

Biology-Psychology Building, Department of Biology, University of Maryland, College Park, Md., USA

(yossy@umd.edu)

The ancestor of cavefish adapted to a completely dark environment by shifting physiological functions. How did cavefish evolve these traits during the adaptation processes? Here we show the physiological and genetic basis of an adaptive behavior, vibration attraction behavior (VAB) in Astyanax mexicanus, which has an eyed surface (surface fish) and a blind cave (cavefish) dwelling forms. VAB is the ability of fish to swim toward the source of a water disturbance in darkness. Quantitative laboratory assays indicate that VAB is common in cavefish but rarely observed and much less robust in surface fish. In competitive prey-capture experiments, surface fish with VAB predominated over those without VAB in darkness but not in light, showing that VAB is beneficial for feeding in the dark. VAB was evoked by vibration stimuli peaking at $35 \mathrm{~Hz}$ and was blocked by the lateral line inhibitors cobalt and gentamicin. The behavior appeared after a numerical increase in superficial neuromast (SN) during development, and 
was significantly reduced by bilateral SN ablation. The significant correlation between VAB and the number of SN supports the conclusion that enhanced SN mediated the evolution of VAB. Mating experiments between surface fish and cavefish suggested that $\mathrm{VAB}$ has a simple genetic basis involving paternal inheritance. Quantitative trait locus (QTL) mapping using 140 F2 offspring showed a single major QTL on the linkage group 17, which accounts for $12 \%$ of the phenotypic variance in VAB. The increase in SN number also had a simple genetic basis (3 QTLs) but none of the SN QTLs was the same as the VAB QTL. Considering the fact that surface fish individuals with VAB do not show an in- crease in SN or tuning at $35 \mathrm{~Hz}$, we propose that the cavefish-type $\mathrm{VAB}$ evolved through sequential SN-independent and SN-dependent steps. As a first step in cavefish evolution, the surface-dwelling ancestor likely used the surface fish-type VAB for initial adaptation to cave environments. In the subsequent step, cavefish with enhanced SN and VAB tuned to $35 \mathrm{~Hz}$ were able to detect prey more efficiently in the dark. This scenario is perhaps one of the ways in which Astyanax became adapted to caves and eventually evolved into cavefish. By the genetic analyses of VAB and SN and the neural tracing of the lateral line system, we will show an evolutionary mechanism for the nervous system. 